\title{
Multiple Dynamic Sinks to Maximize the Lifetime and Conservation of Energy in Wireless Sensor Networks
}

\author{
H. Sivasankari, K. Shaila, K. R. Venugopal, and L. M. Patnaik
}

\begin{abstract}
Wireless sensor networks (WSNs) consist of battery operated tiny sensor nodes and connected in a network for communication. Improving the lifetime of sensor network and energy conservation are the critical issues in WSNs. Nodes closer to the sink node drains their energy faster due to continuous and larger transmission of data towards a sink node. Dynamic Sinks solve the problem of lifetime and energy in WSNs. It moves dynamically to particular positions among the different positions in a predetermined order to collect data from sensor nodes. There is a considerable delay in the case of single mobile sink. In this paper we use the concept of multiple Dynamic sinks to collect data in different zones which in turn coordinate to consolidate the data and complete the process of receiving data from all the sensor nodes. A distributed algorithm synchronizes all dynamic sinks and it is used to reduce delay in consolidation of data and reduces the overall energy consumption. This twin gain increases the lifetime of wireless sensor network and it reduces delay. Simulation results using multiple dynamic synchronized Sinks clearly show that there is an improvement of the lifetime and energy conservation of wireless sensor networks in comparison with single mobile sink and static sink.
\end{abstract}

Index Terms-Energy efficiency, lifetime maximization, multiple dynamic sink, sojourn time and wireless sensor networks.

\section{INTRODUCTION}

Wireless sensor networks (WSNs) use battery operated wireless micro-sensor nodes to collect the information from a geographical field and transmits in multi hop to the sink. Hundreds or thousands of these micro-sensors are deployed to watch the environment and collect data about it. It is used in military to monitor and guide the system of intelligent missiles, detection of attacks by weapons and mass destructions such as chemical, biological and nuclear. WSNs are used in Nature to monitor the fire in the forest, flood detection and habitat exploration of animals and also in the field of health monitoring of patients.

Wireless Sensor Networks with static nodes are deployed randomly to monitor the system. Information generated in a sensor nodes need to reach the destination through a designated gateway. Each node may adjust its power within a certain range that determine that determine the set of possible

Manuscript received November 17, 2011; revised February 15, 2012

H. Sivasankari and K. R. Venugopal are with the Department of Computer Science and Engineering, University Visvesvaraya College of Engineering, Bangalore University, Bangalore 560 001, India.

L. M. Patnaik is with the Indian Institute of Science (IISc), Bangalore, India (e-mail: mailtosankari@yahoo.com). one hop away neighbours. Traffic forwards data to the sink through multiple hops, if the the destination is not in the range. These sensor batteries are impractical to replace or recharge and hence energy of sensor nodes are need to be saved to increase the lifetime of Network. The operational lifetime of a sensor node is in terms of weeks or months. Sensor node spends energy for each process like sensing, transmitting and receiving data. Hence, energy is an important criteria in Wireless Sensor Networks. WSNs have considerable technical challenges in data processing and communication to deal with dynamically changing Energy, Bandwidth, Delay, Sensing and Processing Power. The vital issue in WSNs is to maximize the network operational life. In order to achieve this, it is necessary to minimize the energy utilization of every sensor node.

Efficient routing and data aggregation techniques are used to conserve energy. Another important issue in WSNs is security when it operates in a hostile environment and needs to be protected against intruders. For the small networks, source sensors can directly transmits data to the sink node. For a larger network, multi hop communication is needed to reach the static sink. For real time applications, the sensitive data should reach the sink node without any delay. There are many methods to reduce the distance between the source and the sink. First method is to move the sink node over the entire network to collect the data, the second method is to have multiple static sinks at different locations and third method is to increase the number of mobile sinks. Thus, the distance between the source and sink is reduced.

When an event occurs, immediately the sensor node communicates the information to the sink node. Neighbour nodes of the sink node deplete energy faster due to large and continuous data forwarding towards the sink. Thus, lifetime of the sensor network is reduced even though non-neighbour nodes have enough energy for communication. To overcome this problem Gatzianas et al., [1] have considered the use of single mobile sink. The mobile sink collects the information from the sensor nodes during its round trip time i.e., time during which mobile sink visits entire network in predetermined positions

Motivation: In static sink sensor network, nodes closer to the sink depletes their energy fast, though other nodes in a network have enough amount of energy for communication. The single mobile sink keeps moving to predetermined positions and stays for a specific period of time to collect the data. The sensitive data moving from the source sensors may loose their importance due to the non availability of the single mobile sink. This is due to the delay in the arrival of the single mobile sink to that position. This problem is addressed in this paper by implementing distributed algorithm with 
multiple dynamic sinks and thus sensitive information reaches the sink immediately.

Contribution: The main contribution of this paper is the development of an efficient distributed algorithm using Multiple Dynamic Synchronized Sinks offering an alternative to the single mobile sink. A distributed algorithm for computing the maximum lifetime of a wireless sensor network, which routes data to the nearest dynamic sink by imposing flow conservation to all positions with respect to sinks. An interference-free sensor network with a multiple dynamic synchronized sinks reduces delay, uses less Bandwidth, consume lower energy and it increases the lifetime of WSNs.

Organization: The rest of the paper is organized as follows: Related work and Background work are discussed in Section 2 and Section 3 respectively. System Model and Network Architecture are explained in Section 4. Problem Definition and Mathematical Model is formulated in Section 5. Algorithm is developed in Section 6. Simulation and Performance parameters are analyzed in section 7 . Conclusions are presented in Section 8.

\section{RELATED WORK}

Gatzians et al., [1] addressed the maximization of lifetime of a mobile sink WSNs in-terms of energy constraint. A distributed Synchronous $\varepsilon$-relaxation algorithm based on the sub gradient method is presented to minimize the required time to route data from other nodes of the network to a mobile sink. The system is restricted to semi-deterministic settings resulting in considerable delay.

Michail et al., [2] discussed the routing connection-oriented traffic in wireless sensor networks with energy efficiency. Minimization of data transmission cost with limited bandwidth resources have been considered. Real time constraints in the system and the restriction of nodes to the boundary of location leads to long routing paths between end to end nodes.

Rodoplu et al., [3] proposed a distributed position-based network protocol that optimizes energy consumption in mobile wireless networks for peer-to-peer communications. Network localization, self-reconfiguring protocol, minimum power topology and theory of minimum power stationary networks for data transmission have been considered. Reliability of the output data is the drawback.

Xiao et al., [4] focused on link based optimal routing in wireless data networks. They have exploited a Simultaneous Routing Resource Allocation (SRRA) problem and capacitated multi commodity flow model to describe the data flows in the WSN. Joint link scheduling, routing and power allocations are not emphasized in this work.

Cruz et al., [5] proposed joint routing, link scheduling and power control to support high data rate for broadband network. Shortest path algorithm with link weights and multi-access signal interference model are exploited for optimal routing in the network. Synchronization between transmitters for random channel conditions has not been dealt here.

Kalantri et al., [6] exploited centralized sink network to increase the lifetime of a network. Stavros et al., [7] formulated a distinct calculus of variation technique for optimal placement of nodes in network, which balances the congestion of the shortest data route in sensor networks. Have proposed model for the optimal placement of the node through a scalar nonlinear partial differential equation of calculus. Nodes are placed in the efficient way to avoid bottlenecks. It reduces the congestion and follows the shortest route. It calculates the rate at which the performance of corresponding massive dense networks. It depends on the many factors such as physical layer parameters, MAC, nodes placement and routing protocols. The rate of convergence for any particular interest is not considered. Simulations have been restricted to regular topologies.

Chang et al., [8] considered flow augmentation, flow redirection algorithm to balance the energy among the nodes in proportion to their reserve energy. The robustness of Shortest path routing to maximize the lifetime of a network is not discussed in this work.

Madan et al., [9] formulated a distributed algorithm to compute an optimal routing scheme. The algorithm derived the concept of convex quadratic optimization time constraint to maximize the lifetime of network. They have not considered asynchronous sub-gradient algorithm. Ritesh et al., [10] discussed the mixed integer convex program to maximize the lifetime of network. Non linear class of interference free Time Division Multiple Access for load balancing, Multi hop routing frequency reuse interference mitigation are utilized to increase lifetime of network. The work is restricted for non-distributed low topologies model with lower bound.

Shashidhar et al., [11] proposed a flow based routing protocol to minimize the energy consumption in the sensors of WSNs. Weiwang et al., [12] have used mobile relays to prolong the lifetime of Wireless Sensor Networks. The lifetime of the dense Sensors network with mobile sink and mobile relays are almost same as that of mobile sink.

Tommaro et al., [13] have proposed a sensor-actor co-ordination model to increase the reliability and to reduce the energy consumption of the Wireless Sensor and Actor Networks (WSANs). The collaborative operation of sensors enable the sensing phenomenon of physical nature where the role of actors are used to collect and process the data and take appropriate actions.

Bozidar et al., [14] developed model for power controlled routing protocols to maximize the flow rate. Each node maintains an exclusion region around it. The size of the exclusion region depends on the power constraints of the source of the transmission not on length of the link or the positions of the other nodes. The receiver and the sender adapt the rate of communication according to the amount of noise and interference at the receiver. Routing protocols implemented with the shortest path algorithm either through AODV or DSR is used. The cost of a link is measured and updated during the transmission of every packet.

Chalermek et al., [15] constructed an energy efficient aggregation tree using data centric reinforcement mechanisms. Inefficient paths are pruned using a greedy heuristic approach. They have not considered delay and distinct event delivery ratio. Dasgupta et al., [16] proposed 
energy efficient clustering approach to adapt lifetime maximization of large sensor network. Delay constraints have not been considered in this work.

Sivasankari $\mathrm{H}$ et al., [17] have developed algorithm to reduce energy consumption and to maximize the lifetime of the network. Energy consumption for static and mobile sinks is considered. The lifetime is maximized through the mobile sinks. Energy conservation for all scenarios are discussed

\section{BACKGROUND}

Nodes in a Wireless Sensor Network produce information at a deterministic rate. Sensor Nodes nearer to the static sink, drains energy soon because of large data transmission to the sink node. To increase the lifetime of the sensor network Luo et al., [14] considered a single mobile sink. Each node drains energy as the dynamic sink moves to close to a position of occurrence of an event, spends a specified amount of time to collect the data from the sensors around the location of occurrence of the event. The following conditions are assumed to give feasible solutions to the outgoing links of node to maximize the lifetime. The total expended power should not exceed the initial reserve energy. Peak power transmission constraints are imposed in all the location of sink and time intervals.

A mobile sink can reside in a particular location for nonzero sojourn time and when sojourn time becomes zero, it moves to the next location. In a distributed algorithm each node must store the following information., (i) Sink is distinguished from other nodes by unique node tag identifier, (ii) The maximum rate of information, energy and instantaneous power for each node, (iii) group of variables representing the flow and flow conservation cost which are used in minimum cost flow algorithm, (iv) the outgoing and incoming edges are doubly linked list, (v) a maximum array length to store parent and children of the node, (vi) variables independent of Network size. In the distributed algorithm, the sink in a single round trip time find its neighbours for each location and this information is used to construct a spanning tree. After each round, the sink comes back to the original position. Though the mobile sink increases the lifetime of a network, delay is not considered.

The sink mobility increases the lifetime of sensor network through two methods, (i) based on the relationship between the mobility time scale and (ii) delay time scale. The mobility time scale defined as the time over which the movement of the mobile sink covers a significant portion of a network. The delay time scale is the time over which the mobile sink is not available to the sensor nodes. The following conditions needed to be followed by the routing protocol when data is transferred to the mobile sink. (i) Inform a node when its link gets disconnected with the mobile sink, (ii) The entire network needs to be informed when there is a topology change with respect to the mobile sink, (iii) Packet loss should be minimized when the sink is in motion. (iv) Adaptive algorithm changes the sojourn time of the sink at each location. This algorithm works in two phases (a) initialization and (b) Operation. In the initialization phase, mobile sink visits each anchor points; in operation phase, the sink collects data and power consumption records from all nodes. Though Single Mobile Sink in WSNs increases the lifetime of the network, it has the drawback of delay and single point failure close to a position of occurrence of an event, spends a specified amount of time to collect the data from the sensors around the location of occurrence of the event. The following conditions are assumed to give feasible solutions to the outgoing links of node to maximize the lifetime. The total expended power should not exceed the initial reserve energy. A peak power transmission constraints are imposed in all the location of sink and time intervals. A mobile sink can reside in a particular location for nonzero sojourn time and when sojourn time becomes zero, it moves to the next location.

\section{System Model ANd Network ARChitecture}

\section{A. Definitions}

Mobile Sink: One mobile sink moves to the predetermined positions collecting data from all the neighbouring nodes.

Multiple Dynamic Sinks: More than one mobile or dynamic sink moves to the predetermined positions collecting data from all the neighbouring nodes.

Sojourn Time: The duration of time during which active mobile sink resides in a particular position.

Network Alive: The Network is alive until the sensor can transfer all generated traffic to the nearest sink by satisfying the energy/power and flow conservation constraints.

Energy Consumption: The amount of energy spent by each node in a sensor network for sensing, sending, receiving and processing data.

Li fetime of a Network: The period of time until the first node runs out of energy.

Delay: Time taken by the data to reach the mobile sink node from the source sensor node.

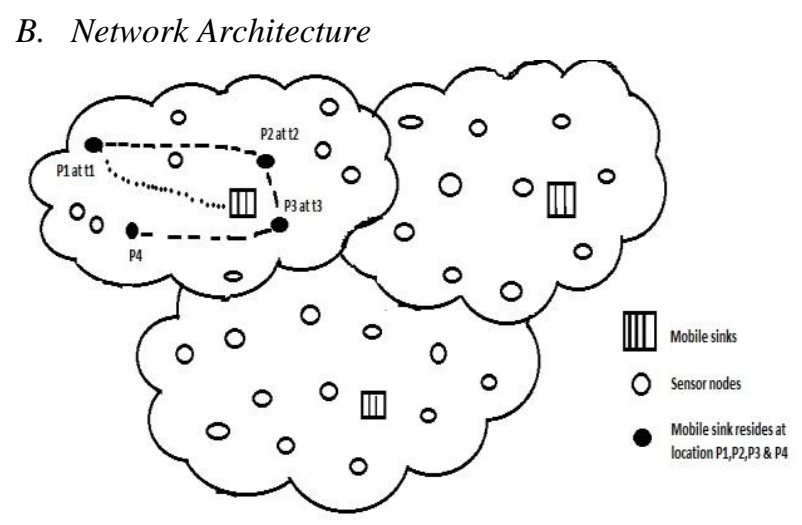

Fig. 1. Basic wireless sensor network architecture with multiple dynamic sinks

The Multiple Dynamic Synchronized Sinks in WSN consist of two types of nodes, (i) Static source sensor nodes sense the event and transmit sensed data, (ii) Mobile sink nodes that move to predetermined positions to collect data from the static Sensor nodes. Multiple dynamic sink nodes can coordinate to consolidate data collected from the static sensor nodes. The Wireless Sensor Network is divided into a number of zones as shown in Fig. 1. The movement of the dynamic sink is restricted to its zone. This technique 
increases the collection of data from ordinary sensor nodes reducing the consumption of energy and delay.

Both these features help in increasing the lifetime of WSNs. When one of the sink fails, the zones are merged. The network still continues to function though at a reduced efficiency, there is increase in delay and is called graceful degradation. The employment of multiple dynamic sink increases reliability and does not allow the WSNs to collapse even with the failure of some Mobile sinks. The multiple dynamic mobile sinks are in continuous communication synchronously and thereby any failure in the sinks will be immediately detected and can be rectified.

\section{Network Model}

Consider a Wireless Sensor Network consisting of battery operated static nodes, which are randomly deployed over a given geographical area. The system model for the mobile sinks or dynamic sink $x_{\mathrm{gm}}$ moves to fixed position $x_{\mathrm{m}}^{\mathrm{p}}$ to avoid early energy dissipation of neighbour nodes of the sink (i.e., as in static sensor network). Each node sensor $i=$ $1, \ldots \ldots, n \in V$ produces a fixed amount of information at rate of $I_{i} \geq 0$. It is assumed that all links between the nodes are in bi-directional. The notations used in this paper are defined in TABLE I. The movement of the sinks to different position creates a subgraph $G(V, L)$. All nodes $i \in V$ except for the sink are equipped with a non-renewable amount of energy $E_{i}>0$. The energy of the sensor is gradually depleted as the nodes participate in routing. Once a node's energy is drained, the node can no longer transmit which leads to network failure. Link capacity $i$ to $j$ is constraint by $\varepsilon_{i}$ i.e

$$
\sum_{j \in S_{i}^{D}} R_{i j} e_{i j} \leq \varepsilon_{i v} \forall i \in \mathbb{N}
$$

For the transmission of data transfer rate in between $i$ and $j$. The minimum power required to transfer data on the link is depend upon the data rate on the link Since the link is bidirectional the transmission power required between $j$ to $i$ is same as that of power needed for $i$ to $j$.

TABLE I: NOTATIONS

\begin{tabular}{|c|c|}
\hline Symbols & Description \\
\hline $\bar{G}$ & Undirected Graph. \\
\hline W & Set of sensor nodes. \\
\hline$S_{i}$ & Edge set or link set. \\
\hline$x_{\mathrm{mx}}$ & $\mathrm{m}^{\mathrm{t} / \mathrm{h}}$ mobile or dynamic sink. \\
\hline$\overline{\mathrm{i}}$ & Source node $i_{x} i=1,2, \ldots \ldots n g$ \\
\hline$S_{i}^{p}$ & $\begin{array}{l}\text { Set of outgoing neighbours of node } \mathbb{L} \text { at sink at position } \\
\text { P. }\end{array}$ \\
\hline$I_{i}$ & Information generated at the node $\bar{i}$. \\
\hline$x_{\mathrm{gx}}^{\mathrm{P}}$ & $p^{\text {th }}$ position of the mobile or dynamic sink $x_{\mathrm{m}} p \in \mathbb{P}_{\mathrm{x}}$ \\
\hline$P$ & Set of mobile sinks positions. \\
\hline$R_{i j}^{p h}$ & $\begin{array}{l}\text { Data transmission rate from node } i \text { to } j \text { while sink stays } \\
\text { at position } p \text { for } k^{\text {th }} \text { iteration. }\end{array}$ \\
\hline$R_{0 j p}^{p}$ & $\begin{array}{l}\text { Data transmission rate from node } i \text { to } j \text { while sink stays } \\
\text { at position } p \text {. }\end{array}$ \\
\hline$t_{x_{m}}^{p i k}$ & $\begin{array}{l}\text { Time for } k^{\text {th }} \text { iteration of } m^{t h} \text { mobile or dynamic sink } \\
\text { at position } p \text {. }\end{array}$ \\
\hline
\end{tabular}

\begin{tabular}{|c|c|}
\hline$e_{i j}^{p h k}$ & $\begin{array}{l}\text { Power needed for data transmission from node } i \text { to } j \\
\text { while sink stays at position } p \text { for } \mathbb{k}^{\text {th }} \text { iteration. }\end{array}$ \\
\hline$e_{\mathrm{d} p}^{p}$ & $\begin{array}{l}\text { Power needed for data transmission from } \\
\text { node } i \text { to } f \text { while sink stays at position } p \text {. }\end{array}$ \\
\hline$t_{g_{m}^{2}}^{P}$ & Sojourn time of the $m^{\text {th }}$ sink at position $p$. \\
\hline$E_{i}$ & Initial Energy of the node. \\
\hline$G^{0}$ & Sub graph $G^{\mathbb{a}} \subset G$ \\
\hline$\theta_{t}$ & Power needed for transmitting one bit of data. \\
\hline$\theta_{\mathrm{Y}}$ & Power needed for receiving one bit of data \\
\hline $\mathrm{k}_{\mathrm{y}}$ & $\mathbb{k}$ bits of data is received. \\
\hline$k_{t}$ & $\mathbb{K}$ bits of data is transmitted \\
\hline$\alpha$ & transmission factor. \\
\hline$\beta$ & Reception factor. \\
\hline$\varepsilon_{\mathbb{i}}$ & Power constraint. \\
\hline$d_{d j}$ & Distance between node i $B_{j} j$. \\
\hline
\end{tabular}

\section{Model Problem Definition AND Mathematical MODEL}

\section{A. Problem Definition}

Given a set of Wireless Sensor Nodes $i \in V$, where $i=$ $1, \ldots \ldots, n$ and set of mobile sinks, $t_{x_{m}}^{p}$ the sojourn time of the $m$ th sink at position $p, t_{x_{m}^{p}}^{p k}$ for a set of iterations where $k=$ $1, \ldots . ., K$. The objectives are

1. To decrease energy consumption and increase the lifetime of the Wireless Sensor Network.

2. To find optimal routing, maintain synchronization between the dynamic sinks, to improve sojourn time and increase the survival time of the network $(T)$. where

$$
\max T=\sum_{1}^{p} \sum_{k=1}^{K} t_{x_{m}}^{p_{k} k}
$$

and can be further reduced to a equivalent form of

$$
\max T=\sum_{1}^{p} t_{a_{m}}^{p}
$$

From Equation 2, we can calculate the maximum lifetime of each dynamic sink at different positions.

\section{B. Assumptions}

1. Sensor nodes are stationary, but the sinks change their positions from time to time with negligible traveling time between two positions. The positions of the sinks can be chosen within a finite set of $x_{\mathrm{m}}^{p} \in P$.

2. A dynamic sink has long range of communication that facilitates to transmit data.

3. Each sensor $i \in V$ produces information at fixed deterministic rate $I_{i} \geq 0$, which is routed in multihops to one of the dynamic sinks $x_{\mathrm{m}}$.

\section{Mathematical Model}

For receiving $k_{y}$ bits/sec, the power consumption at sensor node is $e_{\bar{\gamma}}=\mathbb{k}_{y} \beta$. where, $\beta$ is reception factor indicating the energy consumption per bit. The power needed for transmitting $\mathbb{k}_{\mathrm{t}}$ bits/sec is $e_{\mathrm{t}}=\mathbb{k}_{\mathrm{t}} \propto \mathrm{d}_{\mathrm{ij} \mathrm{j}^{*}}$ where, $\alpha$ is transmission factor indicating the energy consumption per bit and $d_{i j j}$ is the distance between transmitting and receiving node. Therefore, total energy consumption at a node per time unit is 


$$
e_{y}+e_{\mathrm{t}}=k_{y} \beta+k_{\mathrm{t}} \alpha d_{\mathrm{i} j} \otimes e\left(k_{y}+\mathbb{k}_{\mathrm{t}}\right)
$$

where, $e=\beta \approx \alpha d_{i j}$, because energy consumed to transmit a bit is approximately equal to the energy consumed for receiving a bit. From the above discussions, the energy consumption at a sensor node $i$ when the sink sojourn at position $p$ is computed as

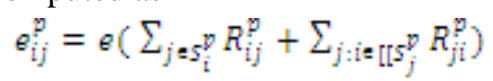

where, $e_{i j}^{g}$ represents the total power needed for data transmission from node $i$ to $j$ while sink stays at position $p$. The energy is calculated through data transmission rate from node $i$ to $j$ and $j$ to $i$ vice versa with respect to the sink's position.

$$
\begin{gathered}
\sum_{1}^{p} \sum_{j=s_{i}^{p}} R_{i j}^{p} e_{i j}^{p} t_{p_{p}^{p}}^{p} \\
\sum_{j=S_{i}^{p}} R_{i j}^{p} e_{i j}^{p} \leq \varepsilon_{i j}, \forall i \\
\sum_{j=S_{i}^{p}} R_{i j}^{p}=
\end{gathered}
$$

The entire geographical deployment area has divided into two zones. In each zone sinks moves to the predefined positions. The dynamic sink moves to different positions to collect the data from the source node. When an event occurs, the sensor senses the data and it forwards the data to the nearest mobile sink positions. If sink is not available in that position then sensors forwards the data to the next available position of the sink. We reduce the Response Time (RT) by introducing the multiple dynamic sinks. The Response Time is computed as

$$
R T=R T_{\text {end }}-R T_{\text {gtart }}
$$

$R T_{\text {start }}$ is the time during which the packet started $R T_{\text {end }}$ is the time during which the packet reached the sink Equation 3 represents the total amount of energy spent at node $i$ and $j$ that depends on the traffic rate on node $i$ and $j$. Equation 4 and 5 explains the energy constraints for communication i.e., energy required for transmitting and receiving data, must not exceed the residual energy of a node. Equation 6 gives the data transmission rate on link $i, j$ i.e., the sum of actual sensed information and traffic rate in the link.

\section{ALGORITHM}

\section{A. Multiple Dynamic Synchronized Sink (MDSS) \\ Distribution Algorithm}

The Multiple Dynamic Synchronized Sink algorithm comprises of two algorithms: MDSS Routing Algorithm and MDSS Iteration Algorithm. In TABLE II the MDSS Distribution Algorithm begins with the selection of dynamic sinks. Each sink moves to a predefined position for a specified period to collect data from each zone. The neighbours of a active sinks are identified by sending the hello packets from all the sensor nodes to the nearest active sink. MDSS algorithm runs for various iterations for different sinks and positions.

MDSS Routing Algorithm selects a minimum distance routing to reduce the energy consumption in the network. When a sensor node has data to forward, it checks for the active sink position and then forwards data. If data transmission time exceeds the sink's sojourn time then forwards data to the next nearest active position of the mobile sink.

The data collection during sojourn time of the mobile sink is referred as iterations (i.e., number of successive transmission). Number of iterations for a particular sink is computed by summing the number of successive transmission during its round trip. The amount of energy dissipated by each node for transmission and reception of data is calculated. If residual energy is equal to zero then network fails. Otherwise, the algorithm runs until one of the node's energy drains to zero in the network. Failure in the sink is detected and repaired as the sinks are synchronized.

\section{IMPLEMENTATION AND PERFORMANCE ANALYSIS}

In the setup of MATLAB simulation, a $100 \mathrm{~m} \times 100 \mathrm{~m}$ region is considered with three sets of network topology with 20, 40 and 80 nodes respectively. The sink is allowed to move over 2, 4 and 8 locations, which are same for all the zones. In all cases, each node has an exogenous rate of $I_{i}=1$.

The flow cost of edge $L(i, j)$ is assumed proportional to $d i$ $j$ the physical distance between the two nodes. The simulation parameters are shown in TABLE III. We consider, as nodes increase in a sensor networks, the number of mobile sinks also increases. The Simulation environment deployed with $8 \times 10$ i.e., 80 nodes. Each node is identified through node identifier as node 1 , node 2 , node 3 etc... The simulation setup is varied for 20, 40, 80 nodes with multiple dynamic sinks. Mobile sink moves in 2, 4, 8 locations and stays for a sojourn time. We observe that, there is a considerable increase in the lifetime of a multiple dynamic sink of Sensor Network in comparison with static and single mobile sink.

TABLE IV, TABLE V and TABLE VI gives the amount of energy residues in each node of 8x10 simulation setup after 914, 1386 and 1646 iterations of Sensor Network with static sink, single mobile sink and multiple dynamic sink respectively. Table 4 shows the residual energy of nodes for single static sink and figure 2 shows the routing tree for the static sink, the

Zeroth row and first column represents node 1, the zeroth node and second column represent node 2 and the first row and first column represent node 11 in this manner nodes 1 to 80 nodes are identified. The amount of energy residues in node 1 is 1.378 J. In Fig. 2, a single static sink is represented as star. Nodes nearer to the sink, continuously participates in routing and thus it drains their energy faster than other nodes present in the deployment.

With a single static sink. The neighbour nodes of static sink is $25,36,66$ and 45 . Nodes among the neighbour node of the continuously participated in data Routing Tree for static sink in 100X100 area with 80 nodes.

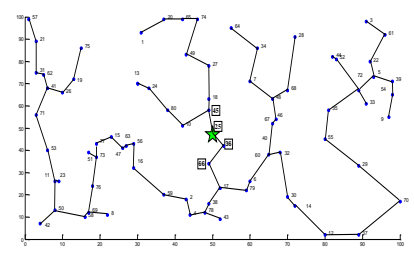

Fig. 2. Routing tree for single static sink in $100 \mathrm{X} 100$ Area with 80 nodes 
TABLE II: MULTIPLE DYNAMIC SYNCHRONISED DISTRIBUTION ALGORITHM. (MDSS)

The subgraph $G{ }^{\prime} \in G$ for all $k$, vectors $V, E, P, I$, initial iteration $k 0$ are taken as input to the algorithm.

Initialize $k=k 0$

Select $x_{m}$ as the number of mobile sinks and predetermine positions as $x_{\mathrm{m}}^{\mathrm{p}}$.

Fix the routing path of each sink $x_{m}$

set arbitrary time $t$;

Begin

.while termination criterion is false do

- for $p=0$ to $|P|-1$ do

Phase 1: MDSS Routing Algorithm

- for $i=1$ to $n^{-} 1 \mathrm{do}$

- for $j=i+1$ to $n$ do

. Calculate the distance between all the nodes with respect to the active position of the sink $x_{\mathrm{ms}}^{\mathrm{F}}$ at time $t$.

total distance $=$ total distance + distance ;

- endfor

- endfor

. compute minimum total distance solve the minimum cost

. flow for subgraph $G^{\prime}$

.determine $t^{p}$;

. if $t_{x_{m}}^{p / 2} \geq$ transmission time then

- Route the data to the nearest active position of the $\operatorname{sink} x_{m}$

- else

- Route the data to the next nearest active position of the

- $\quad \operatorname{sink} x_{m}$

. endif

Phase 2: MDSS Iterations Algorithm

.update $t_{\mathrm{w}_{m}}^{p} \forall(i, j) \in L$

. update from $k$ to $k^{+1}$ for all nodes

- Calculate Energy dissipation as

.Energy dissipation $=$ Transmission Power + Flow Rate

. Calculate Residual Energy of the node

. $E_{i}=$ Residual Energy - Energy dissipation;

. if $E_{i}=0$ then

. return;

. else

. $k=k+1$

.endif

. if sink $x_{m}$ is failed

- then $x_{m+1}$ is made active for that zone.

. endif

. endwhile

End

TABLE III: SIMULATION PARAMETERS

\begin{tabular}{|c|c|}
\hline Parameter type & Test values \\
\hline Number of Nodes & 100 \\
\hline Sink Node & Mote 1 \\
\hline Radio Model & Lossy \\
\hline Multi channel Radio Transceiver & $433 \mathrm{MHZ}$ \\
\hline Sensor type & Temperature, Pressure, Light \\
\hline Outdoor Range & $500 \mathrm{ft}$ \\
\hline Energy Consumption per bit & $60 \mathrm{pJ}$ \\
\hline
\end{tabular}

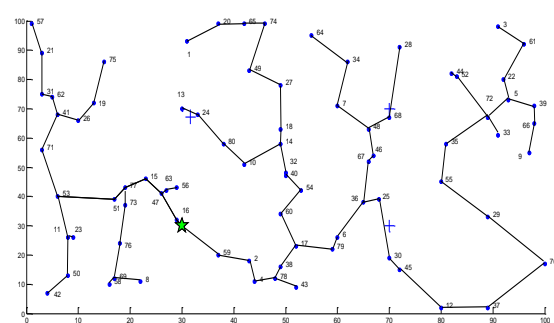

Fig. 3. Routing tree for single mobile sink in $100 \mathrm{X} 100$ area with 80 nodes

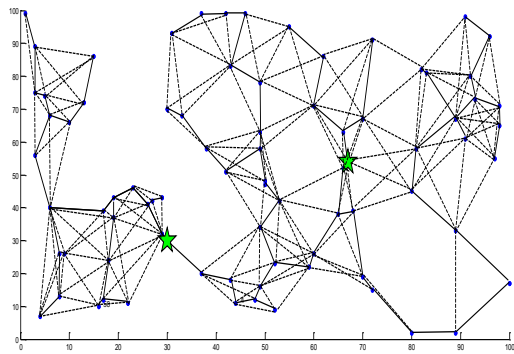

Fig. 4. Complete route tree for multiple dynamic sink in 100X100 Area with 80 nodes

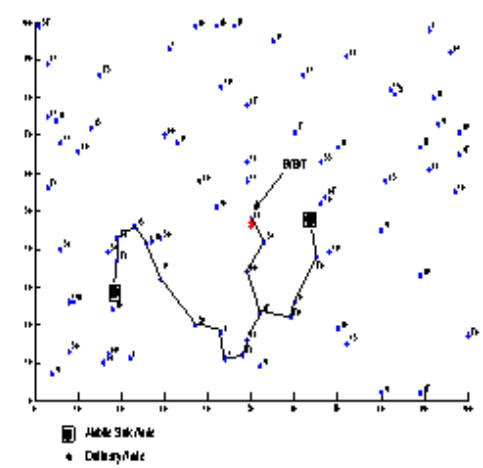

Fig. 5. Comparison of routing between two sinks when a event occurs in the range of both the sinks

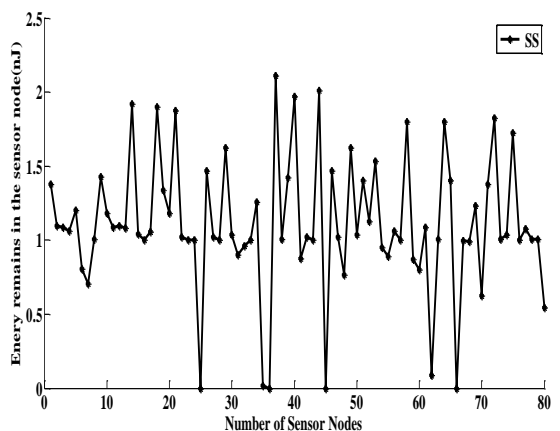

Fig. 6. Residual energy in the SS networks after 914 iterations

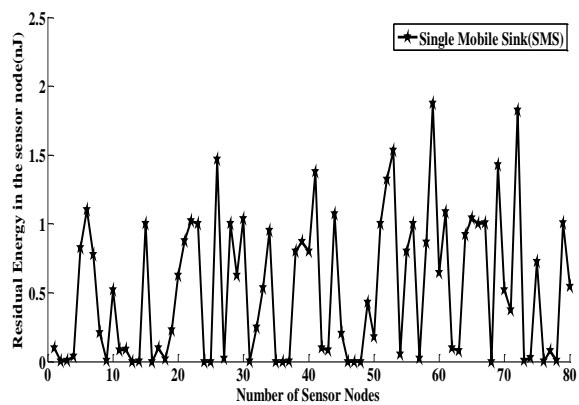

Fig. 7. Residual energy in the SMS networks after 1386 iterations 


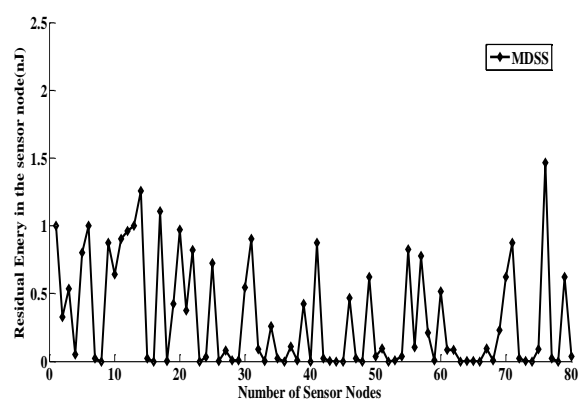

Fig. 8. Residual energy in the MDSS networks after 1646 iterations.

TABLE IV gives the residual energy of the node for the network with the static sink. We observe that nodes 25,36 , 45 and 66 closer to the static sink have zero energy remaining and the network fails after 914 iterations. TABLE V gives the residual energy of the network with single mobile sink. Each node is identified through node identifier as node 1 , node 2 , node 3 etc... The simulation setup is varied for $20,40,80$ nodes with multiple dynamic sinks. Mobile sink moves in 2, 4, 8 locations and stays for a sojourn time.

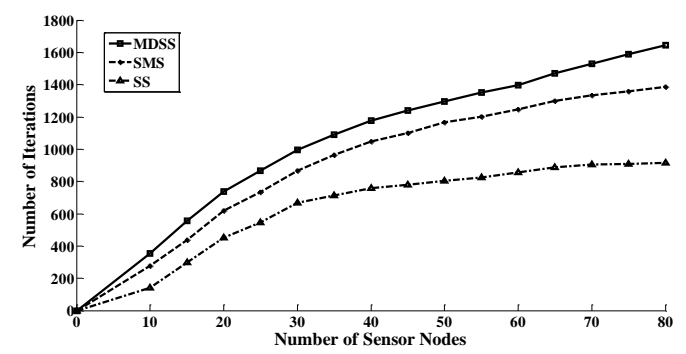

Fig. 9. Comparison between the MDSS, SMS, and SS networks after 914 iterations.

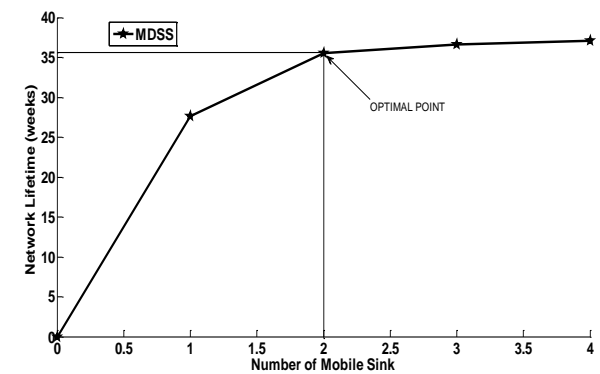

Fig. 10. Optimal number of Sinks for an 80 node network

We observe that, there is a considerable increase in the lifetime of a multiple dynamic sink of Sensor Network in comparison with static and single mobile sink.

TABLE IV: RESIDUAL ENERGY (N J) IN EACH NODE OF 8X10 STATIC SINK WSNS AFTER 914 ITERATIONS.

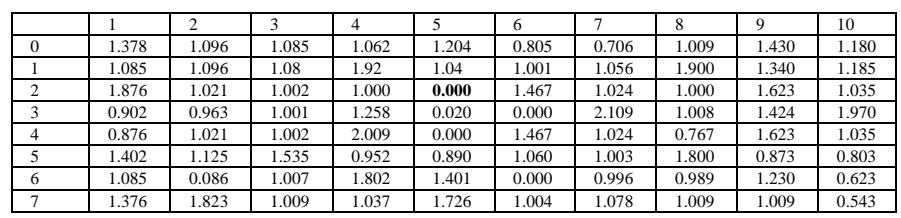

TABLE V: RESIDUAL ENERGY(NJ) IN EACH NODE OF 8X10 SINGLE MOBILE SINK WSNS AFTER 1386 ITERATIONS

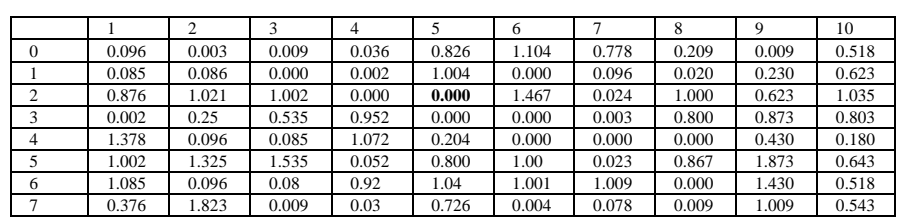

TABLE VI: RESIDUAL ENERGY(N J) IN EACH NODE OF 8X10 MULTIPLE DYNAMIC SINK WSNS AFTER 1646 ITERATIONS

\begin{tabular}{|l|l|l|l|l|l|l|l|l|l|l|}
\hline & 1 & 2 & 3 & 4 & 5 & 6 & 7 & 8 & 9 & 10 \\
\hline 0 & 1.002 & 0.325 & 0.535 & 0.052 & 0.800 & 1.000 & 0.023 & 0.000 & 0.873 & 0.643 \\
\hline 1 & 0.902 & 0.963 & 1.001 & 1.258 & 0.020 & 0.000 & 1.109 & 0.003 & 0.424 & 0.970 \\
\hline 2 & 0.376 & 0.823 & 0.000 & 0.030 & $\mathbf{0 . 7 2 6}$ & 0.004 & 0.078 & 0.009 & 0.009 & 0.543 \\
\hline 3 & 0.902 & 0.090 & 0.001 & 0.258 & 0.020 & 0.000 & 0.109 & 0.008 & 0.424 & 0.000 \\
\hline 4 & 0.876 & 0.021 & 0.002 & 0.000 & 0.000 & 0.467 & 0.024 & 0.000 & 0.623 & 0.035 \\
\hline 5 & 0.096 & 0.000 & 0.009 & 0.036 & 0.826 & 0.104 & 0.778 & 0.209 & 0.009 & 0.518 \\
\hline 6 & 0.085 & 0.086 & 0.000 & 0.002 & 0.004 & 0.000 & 0.096 & 0.009 & 0.230 & 0.623 \\
\hline 7 & 0.876 & 0.021 & 0.002 & 0.000 & 0.090 & 1.467 & 0.024 & 0.000 & 0.623 & 0.035 \\
\hline
\end{tabular}

Fig. 3 displays the routing tree for a single mobile sink, node $13,16,24,25,35,36,46,47,48$ and 69 have zero energy after 1386 iterations where further communication is not possible. TABLE VI represents the residual energy of the network with multiple dynamic sink. In this table node 8,16 , $23,36,39,44,45,48,52,63,66,74$ and 78 has zero energy after 1646 iterations. Fig. 4 shows the complete routing tree for multiple dynamic sinks. This shows that energy at all nodes are used effectively through multiple dynamic sinks, Thus it increases the lifetime of network. Fig. 5 depicts the comparison of routing between two sinks when a event occurs in the range of both sinks. Source sensor node forwards data to the sink which is nearer among the sink nodes.

From the Fig. 6, 7, 8 we infer that, the variance is lower for the network with multiple dynamic sinks. It is observed that all nodes in MDSS network, drain their energy uniformly and thus improves the lifetime of the network. TABLE VII explains the lifetime of the variable networks size. For 10 nodes with static sink network, the lifetime is only 143 iterations which is less than that network with single mobile sink and multiple dynamic sink. Fig. 9 explains the amount of energy residues in each node for the same deployment of $8 \times$ 10 after 914 iterations for Sensor Networks with static sink, single mobile sink and multiple dynamic sink. It is observed that residual energy is higher in each node of that multiple dynamic sink than with the static sink and single mobile sink. The residual energy of nodes in the network with static sink after 914 iterations i.e. at the end of the lifetime is shown in the Fig. 6.

The variance of residue energy in WSN with static sink is 0.2578 . The residual energy of nodes in the network with a single mobile sink after 1386 iterations (Fig. 7). The variance of residue energy in WSN with static sink is 0.2295 . The variance of the residual energy in the network with multiple dynamic sinks is 0.2235 (Fig. 8), which is lower than the network with the static and single mobile sink.

As the number of nodes increase in a given area, the lifetime also increases. The selection of optimal number of mobile sinks depend on the size and density of the network. When the number of mobile sink increases to three, the network lifetime is approximately equals to lifetime of the network with two mobile sinks as shown in Fig. 10. We can conclude that two mobile sinks are optimal for the network with 80 nodes.

Fig. 11 shows the lifetime of the MDSS, SMS and SS. It is observed that the lifetime of MDSS approach is higher than SMS and SS approaches. Fig. 12 explains the amount of energy residues in each node for the same deployment of $8 \times$ 10 after 914 iterations for sensor networks with static sink, single mobile sink and multiple dynamic sink. It is observed that residual energy is higher in each node of dynamic sink 
than with the static sink and single mobile sink. While the lifetime of SS and SMS approach is 914 and 1386 time units, the lifetime of MDSS approach is 1646 units i.e., 56\% more than SS and $28 \%$ more than SMS.

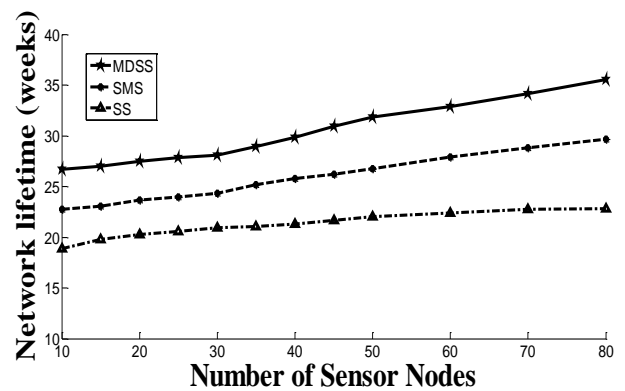

Fig. 11. Comparison of lifetime between the MDSS, SMS and SS networks

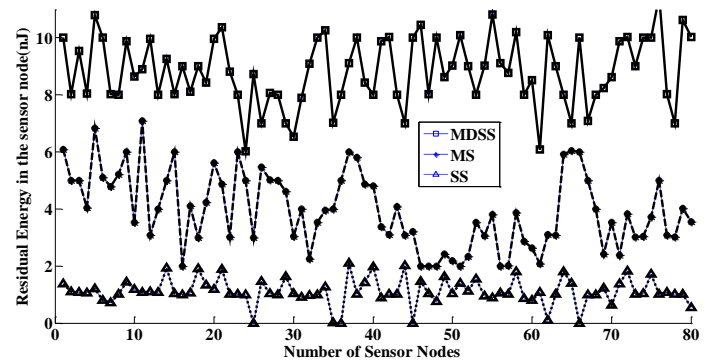

Fig. 12. Comparison between the MDSS, SMS and SS networks 914 iterations.

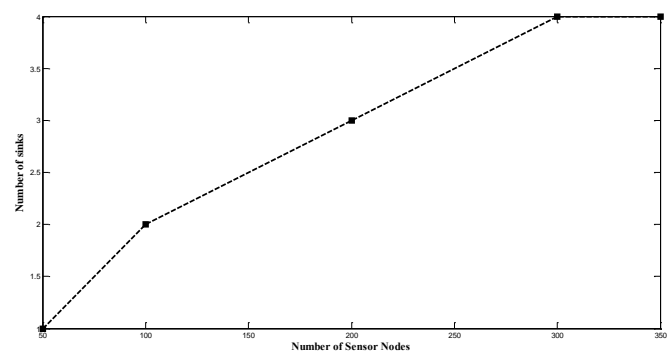

Fig. 13. Number of nodes VS number of sinks

Fig. 13 depicts the number of sinks required for a variable number of sensors. It is observed that two sinks are sufficient for nearly 100 sensor nodes and thereafter there is a linear increase in the requirement of sinks to maintain the desired performance of lifetime and delay. Fig. 14 shows the graph between delay and number of sensor nodes with static sink, single mobile sink and multiple dynamic sinks. The simulation starts with 10 sensor nodes to 80 nodes. Response Time (delay) is calculated as per equation 7 . We observe that there is a considerable reduction in delay for multiple dynamic sink. This reduction of delay is due to less number of hops and reduced distance between the source and the sink.

In the case of Single Static Sink, the average delay is 37 msec for 10 nodes while $30 \mathrm{msec}$ and $25 \mathrm{msec}$ respectively for Single Mobile Sink and Multiple dynamic Synchronized Sink approaches. Thus there is reduction in delay by $50 \%$ in the case MDSS than WSN with Static Sink. As the network density increase, there is gradual reduction in average delay. Though, there is large reduction in delay between SS and SMS, but the reduction is much lower between SMS and MDSS.

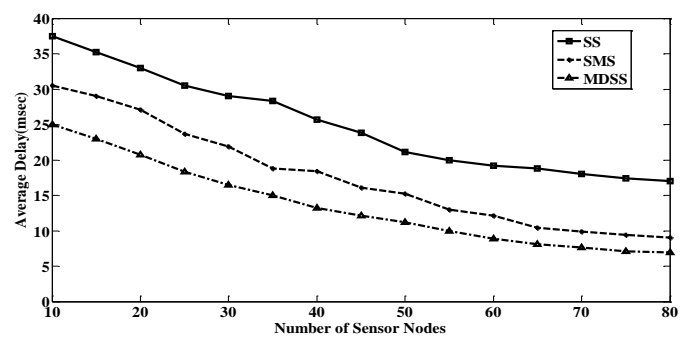

Fig. 14. Number of nodes versus response time (delay)

\section{CONCLUSIONS}

In WSN with a static sink, all source node forwards data towards the sink. In a single mobile sink network, sink moves to pre-determined positions and stays for the sojourn time to collect the data. We propose a distributed algorithm with Multiple dynamic Synchronized Sink to improve the lifetime of the sensor network. A linear program model is proposed to increase the lifetime of the network and to reduce the delay in the transmission of data between the source node and the mobile sink nodes. For the proposed model simulation is carried out for multiple dynamic sink which increases the lifetime by $56 \%$ over single static sink and $28 \%$ over single mobile sink network. During the last iteration of MDSS WSN, the residual energy of all the sensor nodes is almost same which shows that energy drains uniformly and thus increases the lifetime of the network. The proposed MDSS algorithm minimizes the delay in the network at a very small increase in cost of multiple dynamic sink. In future, this can be developed for large scale WSNs including reliability and recovery.

\section{REFERENCES}

[1] M. Gatzianas and L. Georgiadis, "A distributed algorithm for maximum lifetime routing in sensor networks with mobile sink," IEEE Trans. On Wireless Communications, vol. 7, pp. 984-994, 2008.

[2] A. Michail and A. Ephremides, "Energy-efficient routing for connection oriented traffic in wireless Adhoc Networks," IEEE Trans. On Mobile Networks and Applications, vol. 7, pp. 984-994, 2003.

[3] V. Rodoplu and T. Meng, "Minimum energy mobile networks," IEEE ICC, vol. 3, pp. 1633- 1639, 1998.

[4] L. Xiao M. J. and S. Boyd, "Simultaneous routing and resource allocation via dual decomposition," IEEE Transactions Community, volume 52, pp. 1136-1144, 2004

[5] R. Cruz and A. Santhaman, "Optimal routing, link scheduling and power control in multihop wireless networks," in Proc .IEEE INFOCOM, pp. 702-711.

[6] M. Kalantari and M. Shaymam , "Energy efficient routing in wireless sensor networks optimal routing, link scheduling and power control in multihop wireless networks," in Proc .Information Sciences and systems.

[7] J. Chang and L. Tassiulas, "Energy conserving routing in wireless Ad-hoc networks," in Proc. INFOCOM, pp.22-31, 2000.

[8] R. Madan and S. Lall, "Distributed algorithms for maximum lifetime routing in wireless sensor network," IEEE Trans. Wireless Communications, vol. 5, pp.2185-2193, 2006.

[9] S. Ritesh Madan, S. L. Cui, and A. Goldsmith, "Cross- layer design for lifetime maximization in interference- limited wireless networks," in Proc. IEEE INFOCOM, vol. 3, pp.1964-1975, 2005.

[10] V. S. Weiwang and K. C. Chua, "Using mobile relays to prolong the lifetime of wireless sensor networks," in Proc. MOBICO 05, Cologne, Germany. 
[11] M. S. Gandham, R. P. Dawande, and S. Venkatesan, "Energy efficient schemes for WSN with multiple base station." in Proc. IEEE GLOBECOM, vol.1, pp377-381, 2003

[12] W. Wei, V. S, and K. C. Chua, "Using mobile relays to prolong the lifetime of wireless sensor networks," in Proc. MOBICO 05, Cologne, Germany.

[13] T. Melodia, V. G. Daripompilli, and I. F. Akyilidiz, "A distributed co ordination frame work for wireless sensor and actor network," in Proc of MOBIHOC, Urbana- Champaign Illinois, USA, May 2005

[14] C. Intanagonwiwat, D. Estin R. Govindan, and J. Heidemann, "Impact of network density on data aggregation in wireless sensor networks," In Proc 22nd International Conference Discrete Algorithms(SODA '03), January 2003.

[15] K. Dasgupta, K. Kalpakis, and P. Namjoshi, "An efficient clustering-based heuristic for data gathering and aggregation in sensor networks," in Proc, IEEE Press, New York, 2003.

[16] B. Radunovic and J.-Y. L. Boudec, "Optimal power control, scheduling and routing in UWB networks," IEEE Journal on selected areas in Communications, vol. 22, no.7, September 2004.

[17] H. Sivasankari, M. Vallabh, K. Shaila, K. R. Venugopal, and L M Patnaik, "Multiple mobile synchronised sinks(MMSS) for energy efficiency and lifetime maximization in wireless sensor networks," in Proc. 13 ${ }^{\text {th }}$ ICIES-2011, June 2011

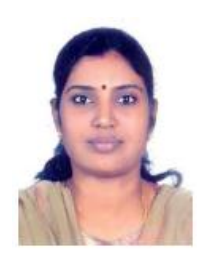

Sivasankari $\mathbf{H}$ is an Associate Professor and Head of the Department of Information Science and Engineering at AMC Engineering College, Bangalore, India. She obtained her B.E in Electronics and Instrumentation Engineering from Bharathiar University, Coimbatore, India and M.E degree in Computer Science and Engineering from Anna University, Chennai, India. She is presently pursuing her Ph. D programme in the area of Wireless Sensor Networks in Jawaharlal Nehru Technological University. Her research interest is in the area of Wireless Sensor Networks and Information Security.

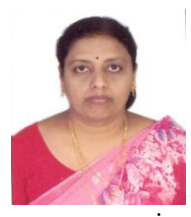

Shaila $\mathbf{K}$ is an Assistant Professor in the Department of Electronics and Communnication Engineering at Vivekananda Institute of Technology, Bangalore, India. She obtained her B.E and M.E degrees in Electronics and communication Engineering from Bangalore Uniuversity. Cuurently she is persuing her $\mathrm{Ph}$. D programme in the area of Security in Sensor Networks in Bangalore University. Her research interest is in the area of Sensor Networks, Adhoc Networks and Image Processing.

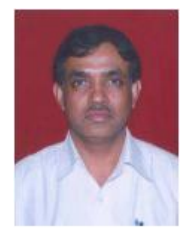

Venugopal $\mathbf{K} \mathbf{R}$ is currently the Principal, University Visvesvaraya College of Engineering, Bangalore University, Bangalore. He obtained his Bachelor of Engineering from University Visvesvaraya College of Engineering. He received his Masters degree in Computer Science and Automation from Indian Institute of Science Bangalore. He was awarded Ph.D. in Economics from

Bangalore University and Ph.D. in Computer Science from Indian Institute of Technology, Madras. He has a distinguished academic career and has degrees in Electronics, Economics, Law, Business Finance, Public Relations, Communications, Industrial Relations, Computer Science and Journalism. He has authored 28 books on Computer Science and Economics, which include Petrodollar and the World Economy, C Aptitude, Mastering C, Microprocessor Programming, Mastering $\mathrm{C}++$,Soft computing for Data Mining etc. During his three decades of service at UVCE he has over 200 research papers to his credit. His research interests include Computer Networks, Parallel and Distributed Systems, Digital Signal Processing and Data Mining.

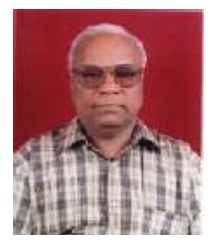

L M Patnaik is a Honorary Professor, Indian Institute of Science, Bangalore. He was former Vice Chancellor, Defense Institute of Advanced Technology, Pune, India. He was a Professor since 1986 with the Department of Computer Science and Automation, Indian Institute of Science, Bangalore. During the past 35 years of his service at the Institute he has over 500 research

publications in refereed International Journals and refereed International Conference Proceedings. He is a Fellow of all the four leading Science and Engineering Academies in India; Fellow of the IEEE and the Academy of Science for the Developing World. He has received twenty national and international awards; notable among them is the IEEE Technical Achievement Award for his significant contributions to High Performance Computing and Soft Computing. His areas of research interest have been parallel and distributed computing, mobile computing, CAD for VLSI circuits, Soft Computing and Computational Neuroscience. 